\title{
Effects of Laughing and Weeping on Mood and Heart Rate Variability
}

\author{
Sokichi Sakuragi ${ }^{1)}$, Yoshiki Sugiyama ${ }^{2)}$ and Kiyomi Takeuchi ${ }^{2)}$ \\ 1) Department of School Nursing and Health Education, Aichi University of Education \\ 2) Department of Health and Psychosocial Medicine, Aichi Medical University School of Medicine
}

\begin{abstract}
We investigated the effects of laughing and weeping induced by watching comedy and tragedy videos on mood and autonomic nervous function. Ten healthy female subjects volunteered for the experiment. Chest electrocardiogram and respiration curve were recorded before, after, and during watching a comedy or a tragedy video. We also asked them to fill out profiles of mood states (POMS) to evaluate their mood states while watching videos. Autonomic nervous function was estimated by spectral analysis of heart rate variability (HRV). All subjects more or less laughed and wept while watching comedy and tragedy videos, respectively. Angerhostility score of the POMS decreased and vigor score increased significantly after watching comedy videos, while depression-dejection score increased significantly after watching tragedy ones. Although both contents tended to increase a low to high frequency component ratio (LF/HF ratio) of $\mathrm{HRV}$, the time course of responses was different. The LF/HF ratio which reflects cardiac sympathovagal balance increased immediately after they started watching comedy videos, and returned to the basal level right after they stopped watching, whereas the LF/ $\mathrm{HF}$ ratio increased gradually to a lesser extent while watching tragedy videos. In contrast, the high-frequency component which reflects cardiac parasympathetic nerve activity gradually decreased while watching both videos but did not return to the basal level after watching tragedy ones. These results suggest that laughing has strong but transient effects on the autonomic nervous system, while weeping or feeling sad has moderate but sustained effects on it. J Physiol Anthropol 21 (3): 159-165, 2002 http:// www.jstage.jst.go.jp/en/
\end{abstract}

Keywords: laughing, weeping, mood state, autonomic nervous system, heart rate variability

\section{Introduction}

Emotions are known to affect the immune system via hypothalamus or other parts of the neuroendocrine system. Several studies have reported beneficial effects of humor or humor-associated laughter that strengthen the immune system such as an increase of natural killer cell activity (Berk et al., 2001; Seaward, 1992). In contrast, some studies have reported negative effects of sorrow, sadness or anxiety on the immune system such as a decrease in the activity of natural killer cells (Ironson et al., 1997; Irwin et al., 1988; Segerstrom et al., 1998). Thus, positive and negative emotions are thought to have positive and negative effects on the immune system, respectively. Emotions are also believed to have some effects on the autonomic nervous system because we sometimes feel palpitation or cold sweating accompanying emotional changes. Numerous studies have reported changes in autonomic nervous function assessed by heart rate variability (HRV) in relation to emotions (Dishman et al., 2000; Friedman and Thayer, 1998a; Friedman and Thayer, 1998b; McCraty et al., 1999; Piccirillo et al., 1997; Pine et al., 1998). However, most of them have described autonomic nervous responses to stresses themselves or stress-associated negative emotions such as anxiety or depression. Laughing and weeping per se are not thought to be stressors but remarkable behavioral expressions associated with pleasant and unpleasant emotions, respectively. We often laugh, for example, when encountering something funny, hear jokes, or watch TV comedy. We also occasionally weep when bereaved, hear sad stories, or see something tragic on TV. However, the effects of laughing or weeping on the autonomic nervous system are not fully understood.

Spectral analysis of HRV is a powerful tool to assess autonomic nervous function because several studies have reported its reliability as a noninvasive index of cardiac autonomic activities (Langewitz et al., 1991; Montano et al., 1994; Pagani et al., 1986; Pagani et al., 1991; Pomeranz et al., 1985). HRV could be divided into two main components by spectral analysis: a high-frequency component (HF component) which reflects parasympathetic nerve activity, and a low-frequency 
component (LF component) which may relate to both sympathetic and parasympathetic nerve activities (Akselrod et al., 1985; Berger et al., 1989; Pomeranz et al., 1985; Pagani et al., 1986; Montano et al., 1994). The profile of mood states (POMS) is also a useful questionnaire to estimate mood states repeatedly. Therefore, to identify the effects of laughing and weeping on the autonomic nervous system and mood states, cardiac autonomic function was assessed by HRV and mood states by POMS questionnaire while watching comedy and tragedy videos.

\section{Methods}

\section{Subjects}

Ten healthy female college students aged 20 to 22 participated in this experiment after given written informed consent. Subjects were told not to eat, drink, or smoke for at least three hours prior to experiments. They were also instructed to go to bed before midnight the night before. The experiments were performed in their follicular phase after the third day of menstration to minimize the influences of a menstrual cycle which could affect mood and autonomic nervous function.

\section{Procedures}

Subjects were instructed to select comedy videos which may make them laugh and tragedy ones which may make them feel sad to tears. The videos were movies or videotaped scenes from TV programs featuring comedy or sad stories. The experiments included two sessions; one for a comedy video and the other for a tragedy one. Each session consisted of three parts, pre-stimulus period for 25 minutes, watching either video for 50 minutes, and post-stimulus period for 25 minutes. The two sessions were conducted at a similar time on two different days out of three consecutive days to avoid the influences of circadian rhythm. The order of the two sessions was counterbalanced to avoid habituation effects.

Each subject sat on a reclining chair in a semi-supine posture. Disc electrodes for chest electrocardiogram (ECG) with CM5 leads and a strain gauge for detecting respiration by impedance method were attached. ECG and respiration curves were recorded during pre-stimulus period for 25 minutes, watching videos for 50 minutes and post-stimulus period for 25 minutes. Data were stored on a DAT recorder (TEAC RD-111T, Tokyo, Japan). Subjects were asked to keep still and remain silent without any big movement of their heads or hands throughout the experiments. They were also asked just to gaze at the screen when the video-player was off during pre- and post-stimulus recordings. After physiological variables were recorded, they were asked to profile their mood states while watching the videos by filling out a
Japanese version of the POMS questionnaire (Yokoyama and Araki, 1994). They were also asked to fill out the POMS questionnaires during their usual mood states in the three consecutive days including two experimental days, to evaluate their basal level of mood states.

\section{Data analysis}

DAT-tapes were played back offline and data were digitized with a sampling frequency of $1 \mathrm{kHz}$ on a personal computer equipped with a 12-bit analoguedigital converter (ADTM-98, Canopus, Kobe, Japan). Experimental sessions were divided into five-minute units for spectral analysis of HRV. After detecting every $R$-wave peak, consecutive R-R intervals on ECG were calculated, and ectopic beats and abrupt discharges in R$\mathrm{R}$ intervals were excluded. Spectral analysis was applied to the time series data of $\mathrm{R}-\mathrm{R}$ intervals for every five minutes, using the maximum entropy method (MemCalc Version 2.5, Suwa Trust) (Ohtomo et al., 1994). After calculating the power spectral density, the magnitude of the power for HRV was obtained by measuring the area under the spectral density curves. The values were divided into two major bands: $\mathrm{LF}(0.04-0.15 \mathrm{~Hz})$ and $\mathrm{HF}$ (0.15-0.4 Hz) domains. The mean amplitudes of each frequency domain were obtained as twice the power magnitude, and the square root thereof. We considered $\mathrm{HF}$ amplitude as an index of cardiac parasympathetic nervous function, and the ratio of $\mathrm{LF}$ amplitude to $\mathrm{HF}$ amplitude (LF/HF amplitude ratio) as that of cardiac sympathovagal balance (Malliani et al., 1991; Pagani et al., 1986). The experimental sessions were divided into four epochs; pre-stimulus (Pre), 1st half of watching a video (1st half), 2nd half of watching a video (2nd half) and post-stimulus (Post). Each epoch had twenty-five minutes. HF and LF amplitude values were normalized to the pre-stimulus mean values and were averaged for ten subjects. The normalized HF amplitudes and LF/HF amplitude ratios were again averaged for each epoch to carry out statistical analysis.

Respiratory frequencies per minute were also assessed every five minutes and were normalized to the prestimulus mean values and averaged for ten subjects.

POMS data were added up for generating six sub-scales, T-A (tension and anxiety), D (depression and dejection), A-H (anger and hostility), V (vigor), F (fatigue) and C (confusion). These added raw scores were converted into T-scores according to the POMS manuals (Yokoyama and Araki, 1994) for parametric statistical analysis. Behavioral responses such as laughing or weeping were confirmed by the examiners. We also averaged raw HRV indices (not normalized data) for each subject while subjects were watching videos and paired with POMS data of the session to calculate Pearson's correlation coefficients between HRV indices and POMS sub-scales. 


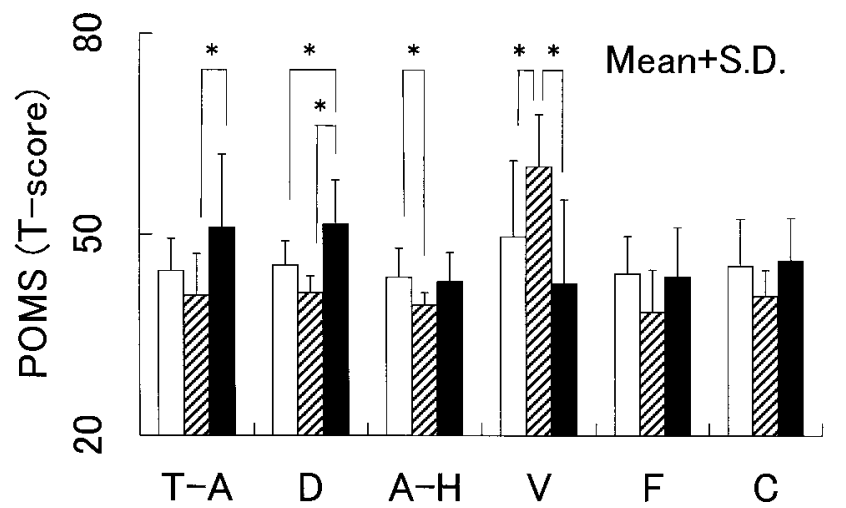

Fig. 1 Profile of Mood States (POMS) T-scores of six sub-scales (TA: Tension-Anxiety, D: Depression-Dejection, A-H: AngerHostility, V: Vigor, F: Fatigue, C: Confusion) at basal state (open bar), while watching comedy videos (striped bar) and tragedy videos (closed bar). A-H score decreased and V score increased significantly while watching comedy videos, whereas D score increased significantly while watching tragedy ones. T-A score while watching tragedy videos was significantly higher than for comedy videos, but was not significant compared to basal one. ${ }^{*} \mathrm{P}<0.05$.

\section{Statistical analysis}

Analysis of variance (ANOVA) with post-hoc comparisons by Fisher's least significant difference was used to clarify the differences in POMS sub-scale T-scores between two experimental sessions and basal state, and the differences in HRV indices during each of the four epochs of the experimental session. Pearson's correlation coefficients were calculated between respiratory frequencies and HRV indices (normalized HF and normalized LF/HF) to evaluate the effects of respiratory frequency on HRV. The correlation coefficients were also calculated between HRV indices (not normalized) and POMS sub-scale T-scores to assess the relationship between mood and autonomic nervous function. Statistical analysis was performed on a personal computer using Statview Ver. 5.0 (HULINKS), and differences with a probability value of less than 0.05 were considered significant.

\section{Results}

All of the subjects laughed and wept while watching comedy and tragedy videos, respectively, although there were some inter-individual varieties in the extent. The A$\mathrm{H}$ score of POMS decreased and the V score increased significantly while watching comedy videos, whereas the D score increased significantly while watching tragedy ones (Fig. 1). The T-A score while watching tragedy videos was significantly higher than for comedy videos, but the difference was not significant compared to the basal level.
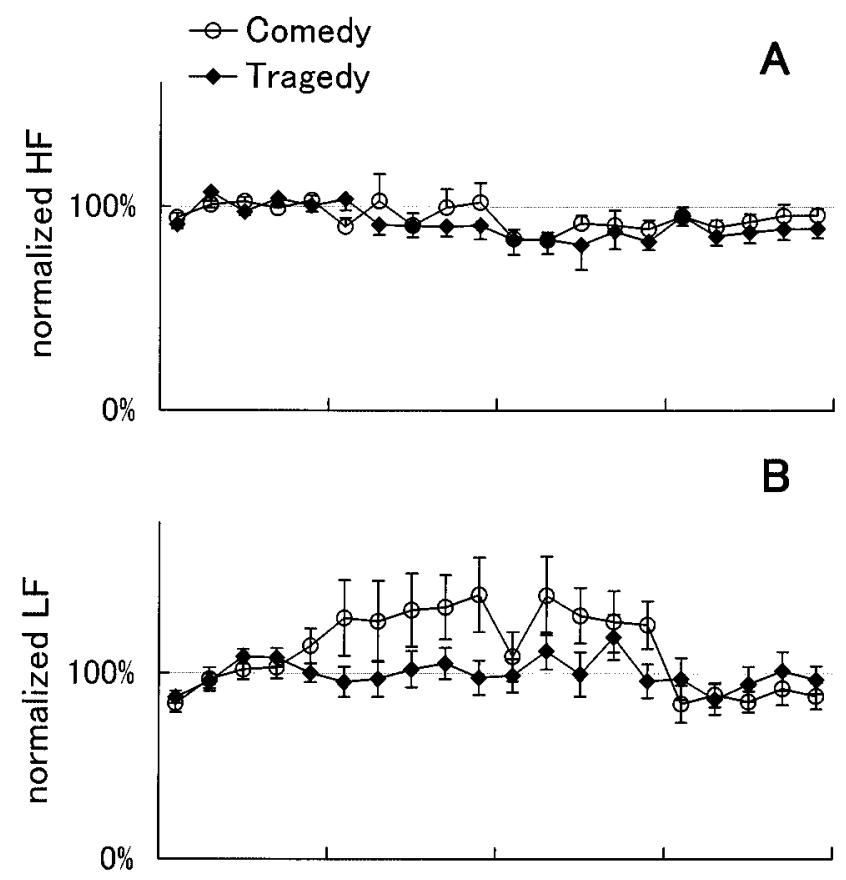

C

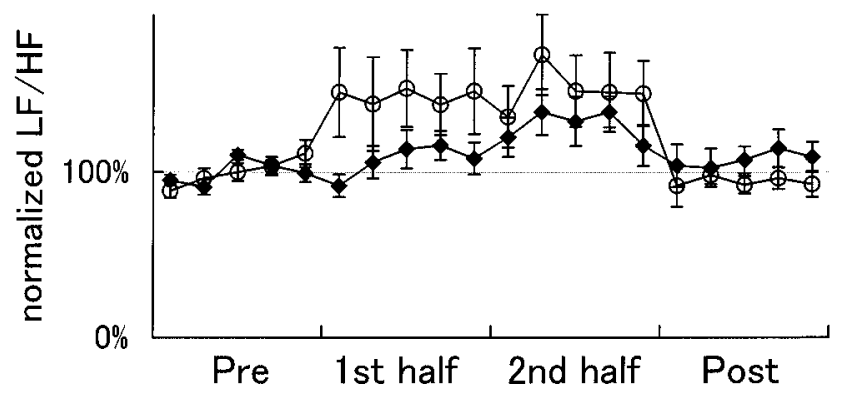

Fig. 2 Normalized HF amplitude (normalized HF), LF amplitude (normalized LF amplitude) and $\mathrm{LF} / \mathrm{HF}$ amplitude ratio (normalized LF/HF) during the sessions. Pre: pre-stimulus (before watching videos), 1st half: during first half of watching videos, 2nd half: during second half of watching videos, Post: post-stimulus (after watching videos). All values are normalized to the pre-stimulus mean values and averaged $(n=10)$. Data are presented as means \pm S.E. A: normalized HF decreased slightly more in the tragedy session than in the comedy one. B: normalized LF increased while watching comedy videos but not for tragedy ones. C: normalized LF/HF increased immediately and gradually while watching comedy and tragedy videos, respectively.

Figure 2 shows normalized HRV indices for every fiveminute unit throughout the session. Each value represents mean \pm S.E. for ten subjects. Normalized HF amplitude (normalized HF) slightly decreased when watching both videos, and seemed to sustain after watching the videos especially tragedy ones (Fig. 2A). Normalized LF amplitude (normalized LF) increased while watching comedy videos and returned to the pre- 


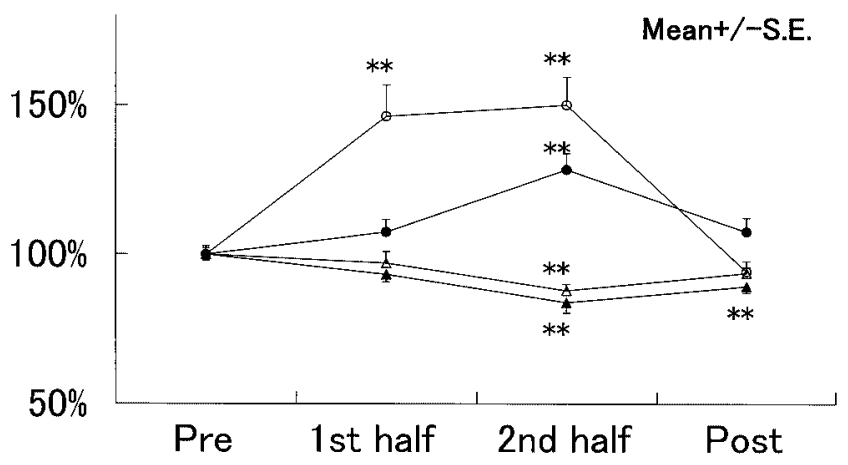

Fig. 3 Average normalized HF and normalized LF/HF during each four epochs in the sessions. Open circle: average normalized LF/ $\mathrm{HF}$ in the comedy session, closed circle: average normalized LF/ $\mathrm{HF}$ in the tragedy session, open triangle: average normalized $\mathrm{HF}$ in the comedy session, closed triangle: average normalized HF in the tragedy session. Significant differences to the pre-values are marked by ** $(\mathrm{P}<0.01$, ANOVA and post-hoc comparison with Fisher's LSD). In the comedy session, normalized LF/HF significantly increased at both the 1 st and 2 nd halves, and immediately returned to the basal level at Post. In the tragedy session, normalized LF/HF increased gradually to a significant level at the 2nd half and returned to a non-significant level at Post. In contrast, normalized HF gradually decreased to a significant level at the 2nd half in both sessions, but did not return to the basal level at Post in the tragedy session.

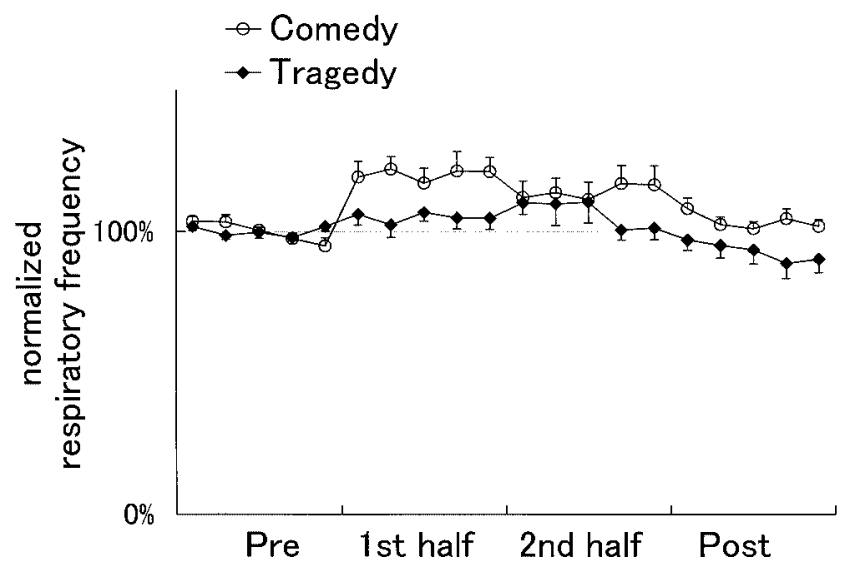

Fig. 4 Normalized respiratory frequency during the sessions. Pre: pre-stimulus (before watching videos), 1st half: first half of watching videos, 2nd half: second half of watching videos, Post: post-stimulus (after watching videos). All values are normalized to the pre-stimulus mean values and averaged $(n=10)$. Data are presented as means \pm S.E. Normalized respiratory frequency increased immediately and gradually while watching comedy and tragedy videos, respectively.

Table 1 Correlation coefficient (r) between HRV indices and POMS sub-scales

\begin{tabular}{lrrrrrr}
\hline & T-A & D & A-H & V & \multicolumn{1}{c}{ F } & C \\
\hline HF amplitude & $0.2904(0.2142)$ & $0.2153(0.3620)$ & $0.0708(0.7669)$ & $0.2686(0.2522)$ & $0.5854(0.0067)^{* *}$ & $0.1527(0.5204)$ \\
LF amplitude & $0.1135(0.6337)$ & $-0.1513(0.5242)$ & $-0.0893(0.7080)$ & $0.2828(0.2271)$ & $0.0790(0.7405)$ & $0.0452(0.8499)$ \\
LF/HF ratio & $-0.1352(0.5699)$ & $-0.3312(0.1537)$ & $-0.1639(0.4899)$ & $0.0523(0.8267)$ & $-0.4150(0.0688)$ & $-0.0753(0.7516)$ \\
\hline
\end{tabular}

Values in parentheses are probabilities. ${ }^{*} \mathrm{P}<0.01$.

stimulus level immediately after watching the videos (Fig. 2B). The normalized $\mathrm{LF} / \mathrm{HF}$ amplitude ratio (normalized LF/HF) increased immediately after they started watching comedy videos and returned abruptly to the basal level after they stopped watching (Fig. 2C). While subjects were watching tragedy videos, their normalized LF/HF gradually increased to a lesser extent compared to that of the comedy session (Fig. 2C). Then, normalized $\mathrm{HF}$ and normalized LF/HF were averaged for each of the four epochs to carry out statistical analysis (Fig. 3). Statistically significant values compared to the pre-stimulus values are marked with asterisks. While subjects were watching comedy videos, their normalized $\mathrm{LF} / \mathrm{HF}$ increased significantly immediately after they started watching and returned to the basal level just after they ceased watching. In the tragedy session, the normalized LF/HF increased gradually to a significant level at 2nd half and returned to a non-significant level after they stopped watching. In contrast, normalized HF component gradually decreased to a significant level at 2nd half while watching both videos, but did not return to the basal level after watching only the tragedy ones.

Respiratory frequencies did not overlap with the frequency domain of LF component, since respiratory frequencies of all subjects were over 10 cycles per minute, i.e., over $0.15 \mathrm{~Hz}$ throughout the experiments. Figure 4 shows that the trend of normalized respiratory frequency in each session resembles that of normalized LF/HF. The normalized respiratory frequency was significantly correlated with normalized LF/HF $(r=0.7073, \mathrm{P}<0.01$ ), but not with normalized HF.

Table 1 shows Pearson's correlation coefficients and pvalues between HRV indices and POMS sub-scales. A significant correlation was observed only between the HF amplitude and POMS F score. 


\section{Discussion}

In the present study, both laughing and weeping increased the $\mathrm{LF} / \mathrm{HF}$ ratio of $\mathrm{HRV}$ and decreased the HF amplitude of HRV, whereas they resulted in different mood states; i.e., the A-H score of POMS decreased and the $\mathrm{V}$ score increased after watching comedy videos, while the D score increased after watching tragedy ones.

Emotions are thought to be related to the autonomic nervous system as well as the immune and endocrine systems. Laughing and weeping are thought to be associated with quite different emotional states. The former expression is considered to accompany a pleasant or positive emotion, the latter with unpleasant or negative one. These opposite aspects were reflected in the POMS scores in our study, that is the A-H (negative mood) score decreased and the $\mathrm{V}$ (positive mood) score increased significantly in the comedy session, while the D (negative mood) score and T-A (negative mood) score in the tragedy session were significantly higher than those in the comedy session (Fig. 1). These opposite aspects were also reflected in the immune system. The beneficial effects of humor or humor-associated laughter and negative effects of sorrow, sadness or anxiety on the immune system have been reported previously (Berk et al., 2001; Ironson et al., 1997; Irwin et al., 1988; Seaward, 1992; Segerstrom et al., 1998). But these opposite aspects were not observed in the autonomic nervous system assessed by spectral analysis of HRV in the present study. In both cases, relative sympathetic predominance with a decrease of cardiac parasympathetic nerve activity was suggested according to the changes in the $\mathrm{LF} / \mathrm{HF}$ ratio and HF amplitude of HRV (Figs. 2, 3). Sohn et al. (2001) indicated the sympathetic involvement in emotional arousal. The results of the present study are consistent with their suggestion.

Laughing and weeping tended to affect HRV in the same direction, though the extents and time courses were somewhat different between them. Several studies suggested the effectiveness of categorizing emotions by mapping them in two-dimensional affective space by their valence (pleasant vs. unpleasant) and arousal (low arousal vs. high arousal or relaxed vs. aroused) to understand the role of the autonomic nervous system in emotion (Lang et al., 1990; Russell MB, 1998). Based on this dimensional analysis of emotion, laughing is considered to be categorized into a pleasant and high arousal dimension. Sadness is usually categorized into an unpleasant and low arousal dimension, but weeping occurs in a situation of extreme sadness. Then, weeping could be categorized into an unpleasant and high arousal dimension. Therefore, the arousal level may be common in laughing and weeping, and high arousal levels induced by laughing and weeping may possibly affect HRV in the same direction.
The LF/HF ratio immediately increased in the comedy session, while gradually increased to a lesser extent in the tragedy session. The HF component gradually decreased to a significant level at the 2nd half epoch while watching both videos, but did not return to the basal level after watching tragedy ones. These results suggest that laughing has strong but transient effects, and weeping or sorrow has slowly rising, mild but sustained effects on autonomic nervous function. Actually, most of the subjects started laughing just after the beginning of the comedy videos, whereas most of them seemed to gradually feel sorrow and started weeping after a while in the tragedy session. These time courses of emotional expressions were almost consistent with those of autonomic nervous function assessed by spectral analysis of HRV in this study. In daily life, we usually laugh immediately, while it takes some time for us to weep. Then, the time course of autonomic nervous function would coincide with that of actual emotional changes. These findings appear to be consistent with the description that pleasant emotions tend to be usually transitory while unpleasant ones can often be persistent (Harrison, 2001).

HRV may not reflect the qualitative changes of mood states, but may possibly reflect the extent of mood changes. In the tragedy session, the $\mathrm{LF} / \mathrm{HF}$ ratio increased and HF amplitude decreased in the stimulus period, and only the HF amplitude remained lowered in the post-stimulus epoch. Relative sympathetic predominance with a decrease of parasympathetic nerve activity was suggested in the stimulus period, and withdrawal of parasympathetic nerve activity was suggested in the post-stimulus epoch. The LF component which corresponds to Mayer wave-related sinus arrhythmia is in relation to periodical blood pressure variation controlled by vasomotor activity and is mediated by both sympathetic and parasympathetic activities. Levenson (1992) suggested the association of some emotion with heart rate change might reflect a close association with a motor program or action tendency which makes significant metabolic demands on the heart. The sympathetic predominance may be related to the peripheral vasoconstrictions mediated by vasomotor sympathetic nerve activity to shift blood supply to the muscles needed for the associated motor program. Mild exercise is known to cause the inhibition of parasympathetic nervous activity, which results in an increase in heart rate (Christensen et al., 1973; Sun et al., 1993). In contrast, moderate or strong exercise activates sympathetic nerve activity with simultaneous withdrawal of parasympathetic nerve activity (Robinson et al., 1966; Yamamoto et al., 1991). Then, high emotional arousal which would trigger a strong motor program might result in activation of sympathetic nervous function with simultaneous inhibition of parasympathetic nervous 
function regardless of whether the mood state is pleasant or unpleasant. And slow or mild emotional arousal which would trigger mild motor program, such as the poststimulus after-effects seen in the tragedy session, might result in inhibition of parasympathetic function without activation of sympathetic function.

We did not instruct subjects to control their respiration because of its difficulty when watching videos. We only monitored the respiratory frequencies to confirm whether the respiratory frequency was in the HF domain. Respiratory frequencies of all subjects were over 10 cycles per minute, i.e., over $0.15 \mathrm{~Hz}$ throughout the experiments. Then the respiratory frequencies were exclusively involved in the HF band and did not overlap the frequency band of the LF component. The respiratory frequency was significantly correlated with the LF/HF ratio, not with the HF component. The HF component, which corresponds to respiratory sinus arrhythmia (RSA), is considered to be affected by tidal volume rather than respiratory frequency (Kobayashi, 1998). Therefore, it was suggested that the change in tidal volume would be in the undetectable range and respiratory frequency would be facilitated by sympathetic activation.

LF amplitude and LF/HF ratio were not significantly correlated with any sub-scales of POMS despite their remarkable changes when watching the videos. Out of six sub-scales of POMS, only F score was positively correlated with HF amplitude. Although the exact meaning of the positive correlation between HF amplitude and F score of POMS is not known, one possible explanation might be that our arousal level may become lower when we are fatigued, then the parasympathetic nervous function can possibly get activated, resulting in an increase in the HF amplitude of HRV. Thus, activation of the parasympathetic nervous function may induce restoration of the vigor.

In this study, we only examined the acute effects of laughing or weeping on the autonomic nervous system. When we consider their use in stress coping, we should study the chronic effects of laughing or weeping on the autonomic nervous system.

Acknowledgement $\mathrm{We}$ appreciate the generous cooperation of I. Sugiyama, K. Takeuchi, S. Fujiyoshi, E. Matsuzawa and A. Yamauchi in the study.

\section{References}

Akselrod S, Gordon D, Madwed JB, Snidman NC, Shannon DC, Cohen RJ (1985) Hemodynamic regulation: investigation by spectral analysis. Am J Physiol 249 (Heart Circ Physiol 18): H867-H875

Berger RD, Saul JP, Cohen RJ (1989) Transfer function analysis of autonomic regulation. I. Canine atrial rate response. Am J Physiol 256 (Heart Circ Physiol 25): H142-H152

Berk LS, Felton DL, Tan SA, Bittman BB, Westengard J (2001) Modulation of neuroendocrine parameters during the eustress of humor-associated mirthful laughter. Altern Ther Health Med 7: 62-72, 74-76

Christensen NJ, Brandsborg O (1973) The relationship between plasma catecholamine concentration and pulse rate during exercise and standing. Eur J Clin Invest 3: 299-306

Dishman RK, Nakamura Y, Garcia ME, Thompson RW, Dunn AL, Blair SN (2000) Heart rate variability, trait anxiety, and perceived stress among physically fit men and women. Int J Psychophysiol 37: 121-133

Friedman BH, Thayer JF (1998a) Anxiety and autonomic flexibility: a cardiovascular approach. Biol Psychol 49: 303-323

Friedman BH, Thayer JF (1998b) Autonomic balance revisited: panic anxiety and heart rate variability. $\mathrm{J}$ Psychosom Res 44: 131-151

Harrison GA (2001) Comparative stress in human societies. J Physiol Anthropol 20: 49-53

Ironson G, Wynings C, Schneiderman N, Baum A, Rodriguez M, Greenwood D, Benight C, Antoni M, LaPerriere A, Huang HS, Klimas N, Fletcher MA (1997) Posttraumatic stress symptoms, intrusive thoughts, loss, and immune function after Hurricane Andrew. Psychosom Med 59: 128-141

Irwin M, Daniels M, Risch SC, Bloom E, Weiner H (1988) Plasma cortisol and natural killer cell activity during bereavement. Biol Psychiatry 24: 173-178

Kobayashi H (1998) Normalization of respiratory sinus arrhythmia by factoring in tidal volume. Appl Human Sci 17: 207-213

Lang PJ, Bradley MM, Cuthbert BN (1990) Emotion, attention, and the startle reflex. Psychol Rev 97: 377395

Langewitz W, Rüddel H, Schächinger H, Lepper W, Mulder LJM, Veldman JHP, van Roon A (1991) Changes in sympathetic parasympathetic cardiac activation during mental load: an assessment by spectral analysis of heart rate variability. Homeostasis 33: 23-33

Levenson RW (1992) Autonomic nervous system differences among emotions. Psychol Sci 3: 23-27

Malliani A, Pagani M, Lombardi F, Cerutti S (1991) Cardiovascular neural regulation explored in the frequency domain. Circulation 84: 482-492

McCraty R, Atkinson M, Tomasino D, Goelitz J, Mayrovitz HN (1999) The impact of an emotional selfmanagement skills course on psychosocial functioning and autonomic recovery to stress in middle school children. Integr Physiol Behav Sci 34: 248-268

Montano N, Ruscone TG, Porta A, Lombardi F, Pagani M, Malliani A (1994) Power spectrum analysis of heart rate variability to assess the changes in sympathovagal 
balance during graded orthostatic tilt. Circulation 90 : 1826-1831

Ohtomo N, Terachi S, Tanaka Y, Tokiwano K, Kaneko N (1994) New method of time series analysis and its application to wolf's sunspot number data. Jpn J Appl Phys 33: 2821-2831

Pagani M, Lombardi F, Guzzetti S, Rimoldi O, Furlen R, Pizzinelli P, Sandrone G, Malfatto G, Dell'Orto S, Piccaluga E, Turiel M, Baselli G, Cerutti S, Malliani A (1986) Power spectral analysis of heart rate and arterial pressure variabilities as a marker of sympathovagal interaction in man and conscious dog. Circ Res 59: 171-192

Pagani M, Rimoldi O, Pizzinelli P, Furlen R, Crivellaro W, Liberati D, Cerutti S, Malliani A (1991) Assessment of the neural control of the circulation during physiological stress. J Auton Nerv Syst 35: 33-42

Piccirillo G, Elvira S, Bucca C, Viola E, Cacciafesta M, Marigliano V (1997) Abnormal passive head-up tilt test in subjects with symptoms of anxiety power spectral analysis study of heart rate and blood pressure. Int $\mathrm{J}$ Cardiol 60: 121-131

Pine DS, Wasserman GA, Miller L, Coplan JD, Bagiella E, Kovelenku P, Myers MM, Sloan RP (1998) Heart rate variability and psychopathology in urban boys at risk for delinquency. Psychophysiology 35: 521-529

Pomeranz B, Macauly RJB, Caudil MA, Kutz I, Adam D, Gordon D, Kilborn KM, Barger AC, Shannon DC, Cohen RJ, Benson H (1985) Assessment of autonomic function in humans by heart rate spectral analysis. Am J Physiol
248: 151-153

Robinson BF, Epstein SE, Beiser GD, Braunwald E (1966) Control of heart rate by the autonomic nervous system. Circ Res 19: 400-411

Russell MB (1998) Physiologic measures of emotion. J Clin Neurophysiol 15: 388-396

Seaward BL (1992) Humor's healing potential. Health Prog 73: 66-70

Segerstrom SC, Solomon GF, Kemeny ME, Fahey JL (1998) Relationship of worry to immune sequelae of the Northridge Earthquake. J Behav Med 21: 433-450

Sohn JH, Sokhadze E, Watanuki S (2001) Electrodermal and cardiovascular manifestations of emotions in children. J Physiol Anthropol 20: 233-239

Sun JC, Eiken O, Mekjavic IB (1993) Autonomic nervous control of heart rate during blood-flow restricted exercise in man. Eur J Appl Physiol 66: 202-206

Yamamoto Y, Hughson RL, Peterson JC (1991) Autonomic control of heart rate during exercise studied by heart rate variability spectral analysis. J Appl Physiol 71: 1136-1142

Yokoyama K, Araki S (1994) POMS Japanese manual. Kaneko Syobo, Tokyo (in Japanese)

Received: July 23, 2001

Accepted: March 13, 2002

Correspondence to: Sokichi Sakuragi, Department of School Nursing and Health Education, Aichi University of Education, Hirosawa 1, Igaya-cho, Kariya 448-8542, Japan e-mail: ssakurag@auecc.aichi-edu.ac.jp 